\title{
The Continuous Galerkin Finite Element Method Is Not Naturally Consistent with the Second Law of Thermodynamics
}

\author{
Alejandro C. Limache ${ }^{a, *}$, Hugo Aimar ${ }^{\mathrm{a}}$ \\ ${ }^{a}$ Instituto de Matemática Aplicada del Litoral (IMAL), Santa Fe, Argentina
}

\begin{abstract}
It is well known that the Continuous Galerkin Finite Element (CGFE) method is globally consistent with respect to the first law of thermodynamics. This means that, for any mesh, all obtained discrete solutions will conserve total energy. One might expect, that the method is, also, globally consistent with respect to the second law of thermodynamics. In this paper, we formally study if such conjecture is true. The heat conduction equation is used as the physical model for this analysis. In the present study it is proved that the conjecture is false: at least, for standard piecewise linear (1D and 2D) elements, the CGFE method is not always globally consistent with respect to the second law of thermodynamics. In other words, some obtained discrete solutions can violate the global postulate of the second law, which asserts that total entropy can never decrease.
\end{abstract}

Keywords: finite element method; second law of thermodynamics; heat equation; entropy

\section{Introduction}

Some physical principles, originally present in a continuous model, can get lost due to the particularities of the discretization procedure leading to a numerical scheme. Many problems, such as, instabilities, convergence failures or even undetected non-physical solutions can arise because of such loss [1], [2]. Consequently, it might be critical to assess if commonly used

\footnotetext{
${ }^{*}$ Dr. A. Limache, IMAL-CONICET, Guemes 3450, Santa Fe, ARGENTINA $U R L$ : alejandrolimache@gmail.com (Alejandro C. Limache )
} 
discretization methods preserve fundamental physical principles. The Continuous Galerkin Finite Element (CGFE) method is of the most renowned discretization methods, particularly, in physical applications. In this sense, it is of interest to question if such method preserves the two fundamental laws of thermodynamics. On this regard, it is already known that such method is globally consistent with respect to the first law [3]. This means that, for any mesh, all obtained CGFE solutions conserve total energy. However, nothing has been said about the global consistency of the method with respect to the second law. By analogy, with the first law, one might expect that the CGFE method is, also, globally consistent with the second law of thermodynamics. In other words, one would expect that all CGFE solutions satisfy the global statement of the second law, at all times, independently of the mesh. However, a recently appeared paper [4] has brought attention to the fact that some finite element discretizations can generate discrete reversed non-physical nodal heat-fluxes where heat, non-physically, goes from colder to warmer nodal points. These experiments prove that some finite element solutions violate, nodally, Clausius's Postulate of the second law of thermodynamics. The observations presented in [4] might be argued to be merely qualitative since the nodes per se have not a direct meaning in a weak/integral framework, as the one of finite elements. It is then necessary to formally prove if the hypothesis of global consistency of CGFE discretizations with respect to the second law, is true or false. This is the purpose of the present article. The unsteady heat equation will be used for the analysis.

The paper is organized as follows, in Section 2, the necessary thermodynamic theoretical background is introduced. There, the global forms of the first and the second law of thermodynamics are given. In Section 3, the unsteady heat equation is introduced, with their respective initial conditions and boundary conditions. After presenting the partial differential equation, a thermodynamic analysis of the exact solutions is perfomed in subsection 3.2. There, it is proved that all exact solutions of the heat equation satisfy the global statements of the first and the second law of thermodynamics. This result is known but serves for comparison with the forthcoming analysis of thermodynamic consistency of the discrete CGFE solutions. The CGFE discretization method is introduced at the beginning of Section 4, and applied to the model heat equation, leading to a discretized heat equation problem. Subsection 4.2 contains the thermodynamic analysis of the resulting CGFE discrete solutions with respect to the first law and the second law of thermodynamics. In subsection 4.2.1, the global consistency of CGFE solutions with respect to the first law is proved. This result is known but it serves as a preliminary setup for the thermodynamic analysis with respect 
to the second law. In subsection 4.2.2, the global consistency of CGFE solutions with respect to the second law is studied. After some general results, presented as lemmmas, it is proved, by means of examples that, for standard piecewise linear elements in $1 \mathrm{D}$ and $2 \mathrm{D}$, the CGFE method is not globally consistent with respect to the second law of thermodynamics. The examples prove that the CGFE method can generate discrete solutions that violate the condition that total entropy can never decrease in an isolated system.

Notation: Given an arbitrary field $f(\boldsymbol{x}, t)$ function of position $\boldsymbol{x}$ and time $t$, the partial derivative with respect to time will be denoted as $\dot{f}(\boldsymbol{x}, t)$, so $\dot{f}(\boldsymbol{x}, t) \stackrel{\circ}{=} \frac{\partial f(\boldsymbol{x}, t)}{\partial t}$. Similarly, given an arbitrary function $g=g(t)$ of time $t$,

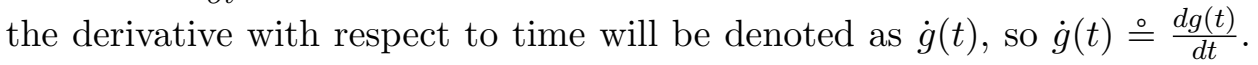
Whenever there is no risk of confusion, the explicit dependence on $\boldsymbol{x}$ and $t$ will be dropped, so, for example, $\dot{f}(\boldsymbol{x}, t)$ and $\dot{g}(t)$, will be simply written as $\dot{f}$ and $\dot{g}$, respectively.

\section{The First and the Second Laws of Thermodynamics}

\subsection{General Forms}

The laws of thermodynamics can be seen as a set of two requirements that physical models should fullfill in order to correctly model time evolution of physical phenomena. Let us recall the main concepts using the modern theory of thermodynamics of continua. In such framework, consider an fixed material body $B$ of uniform density $\rho$, ocuppying an arbitrary spatial domain $\Omega$. For future use, we will denote the boundary of $\Omega$ by $\partial \Omega$ and by $\mathbf{n}$ the outward point unit normal to $\partial \Omega$. Now, given an initial state at $t=0$, the physical variables evolve as function of time $t$ in the whole body's domain $\Omega$. Assume that an arbitrary mathemathical model provides to us the values of the scalar and vector fields $\mathcal{T}(\boldsymbol{x}, t), \mathfrak{e}(\boldsymbol{x}, t), \mathfrak{s}(\boldsymbol{x}, t)$ and $\mathfrak{q}(\boldsymbol{x}, t)$, which model the main thermodynamic physical variables: temperature, especific energy, specific entropy and the heat flux vector, respectively, $\forall \boldsymbol{x} \in \Omega, \forall t \geq 0$. Let us denote by $\Pi$, the tuple that contains the four fundamental thermodynamic fields, ordered as indicated below:

$$
\Pi=[\mathcal{T}, \mathfrak{e}, \mathfrak{s}, \mathfrak{q}]
$$

With the above definitions, the two laws of thermodynamics can be stated as a set of restrictions on the tuple $\Pi$. The first law imposes a restriction of 
conservation of energy, and its differential and integral forms are given by:

$$
\left\{\begin{array}{lll} 
& \text { First Law } \\
\text { diff. form } & \rho \dot{\mathfrak{e}}=-\nabla \cdot \mathfrak{q} \\
\text { integral form } & \int_{V} \rho \dot{\mathfrak{e}} d v=-\int_{\partial V} \mathfrak{q} \cdot \mathbf{n} d \sigma, V \subseteq \Omega(b) \\
\text { global form } & \int_{\Omega} \rho \dot{\mathfrak{e}} d v=-\int_{\partial \Omega} \mathfrak{q} \cdot \mathbf{n} d \sigma
\end{array}\right.
$$

On the other hand, the second law imposes an inequality restriction on entropy growth (known as Clausius-Duhem inequality), and its differential and integral forms are given by:

$$
\left\{\begin{array}{lll} 
& \text { Second Law } \\
\text { diff. form } & \rho \dot{\mathfrak{s}} \geqslant-\nabla \cdot\left(\frac{\mathfrak{q}}{\mathcal{T}}\right) & (a) \\
\text { integral form } & \int_{V} \rho \dot{\mathfrak{s}} d v \geqslant-\int_{\partial V} \frac{1}{\mathcal{T}} \mathfrak{q} \cdot \mathbf{n} d \sigma, V \subseteq \Omega \\
\text { global form } & \int_{\Omega} \rho \dot{\mathfrak{s}} d v \geqslant-\int_{\partial \Omega} \frac{1}{\mathcal{T}} \mathfrak{q} \cdot \mathbf{n} d \sigma
\end{array}\right.
$$

The above formulas can be found in standard textbooks, such as, [5], [6]. Note that in equations above, we have also introduced the global forms of the laws of thermodynamics. The global forms are particular cases of the integral forms when volume $V$ is chosen to be the whole volume $\Omega$.

\subsection{Global forms for Isolated Domains}

In this paper, we are going to center the analysis on whether or not the global forms are satisfied by given thermodynamic tuples $\Pi$. In particular, we are going to explore the cases where our system (i.e. the material domain $\Omega$ ) is fully isolated from external heat. Under this situation, also known as adiabatic condition, the following boundary condition holds:

$$
\text { full isolation b.c. } \Rightarrow \mathbf{q} \cdot \mathbf{n}=0 \text {, on } \partial \Omega
$$

Using this boundary condition, the global forms of the laws of thermodynamics given in Eqs. (2) (d) and (3) (d) become:

$$
\begin{cases}\text { Isolated Domains } & \\ & \text { First Law } \\ \text { global form } & \int_{\Omega} \rho \dot{\mathfrak{\varepsilon}} d v=0 \quad(a) \\ & \text { Second Law } \\ \text { global form } & \int_{\Omega} \rho \dot{\mathfrak{s}} d v \geqslant 0 \quad(b)\end{cases}
$$

Then, given an arbitrary tuple $\Pi$, we will say that $\Pi$ is globally consistent with respect to the first law if it satisfies Eq. (5) (a). Similarly, $\Pi$ will be globally consistent with respect to the second law if it satisfies Eq. (5)(b). 
Note that, for isolated systems, satisfaction of the global statements of the first and second law of thermodynamics express that total energy $\mathcal{E}(t)=$ $\int_{\Omega} \rho \mathfrak{e}(\boldsymbol{x}, t) d v$ must remain constant $(\dot{\mathcal{E}}(t)=0)$ and that total entropy $\mathcal{S}(t)=$ $\int_{\Omega} \rho \mathfrak{s}(\boldsymbol{x}, t) d v$ must not decrease $(\dot{\mathcal{S}}(t) \geqslant 0)$, respectively. This concepts will be used throughout the rest of the article.

\subsection{Linear Heat Conducting Material}

The laws of thermodynamics presented in the previous subsections are general, nothing has been said about the particular constitutive properties of the material occupying the domain $\Omega$. For future use, let us consider here, the particular case, where the body is made of a linear heat conducting material. A linear heat conducting material is a material with the following constitutive properties: (i) specific energy $\mathfrak{e}$ is proporcional to temperature and (ii) heat flux $\mathbf{q}$ is proportional to minus the temperature gradient. In other words, given an arbitrary temperature field $\mathcal{T}$, specific energy and heat flux are given by the following equations:

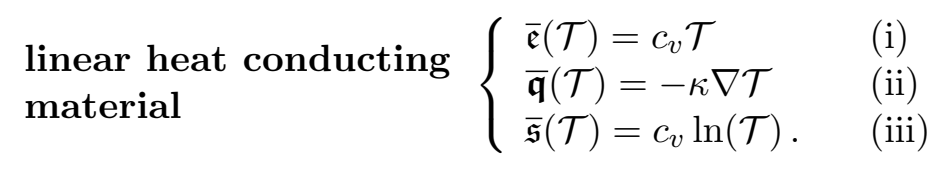

where $c_{v}$ and $\kappa$ are given positive constants. Note that in the constitutive properties given in Eq. (6), we have added the expression of the entropy function $\overline{\mathfrak{s}}$ which provides the value of specific entropy $\mathfrak{s}$ given the value of temperature $\mathcal{T}$. Entropy is crucial to test consistency with respect to the second law of thermodynamics. The entropy function $\overline{\mathfrak{s}}$ is determined under the hypotheses of heat conduction in a rigid material domain. In such case, $\overline{\mathfrak{e}}$ and $\overline{\mathfrak{s}}$ are functions of temperature only, and they must satisfy Gibb's relationship:

$$
\frac{d \overline{\mathfrak{s}}(\mathcal{T})}{d \mathcal{T}}=\frac{1}{\mathcal{T}} \frac{d \overline{\mathfrak{e}}(\mathcal{T})}{d \mathcal{T}}
$$

Since, $\overline{\mathfrak{e}}(\mathcal{T})=c_{v} \mathcal{T}$, Eq. (7), leads to $\overline{\mathfrak{s}}(\mathcal{T})=c_{v} \ln (\mathcal{T})$, which is the function presented in Eq. (6)(iii). In this case, the following equations are valid for time rates:

$$
\begin{cases}\frac{\partial \overline{\mathfrak{e}}(\mathcal{T})}{\partial t}=c_{v} \dot{\mathcal{T}} & (a) \\ \frac{\partial \overline{\mathfrak{s}}(\mathcal{T})}{\partial t}=c_{v} \dot{\mathcal{T}} & (b)\end{cases}
$$

Eqs. (6) provide the values of specific energy energy $\mathfrak{e}$, heat flux $\mathfrak{q}$ and specific entropy $\mathfrak{s}$ given the temperature field $\mathcal{T}$. Then, it follows that the 
thermodynamic tuple $\Pi=[\mathcal{T}, \mathfrak{e}, \mathfrak{s}, \mathfrak{q}]$ associated to any linear heat conduction material must have the following form:

$$
\bar{\Pi}(\mathcal{T})=[\mathcal{T}, \overline{\mathfrak{e}}(\mathcal{T}), \overline{\mathfrak{s}}(\mathcal{T}), \overline{\mathfrak{q}}(\mathcal{T})]
$$

With the above formulas, we have ready the framework to analyze the thermodynamic consistency of exact and numerical solutions of the heat equation. This will be done in the next two following sections.

\section{Thermodynamic Consistency Analysis of the Heat Equation}

\subsection{The Unsteady Heat Equation}

Let us consider, the temperature solutions $T(\boldsymbol{x}, t)$ of the unsteady heat equation in a domain $\Omega$ with Neuman boundary conditions, posed as the following problem:

$$
(\mathbf{P})\left\{\begin{array}{lll}
\text { Find } T(\boldsymbol{x}, t), \text { such that } & \\
\rho c_{v} \dot{T}=\nabla \cdot(\kappa \nabla T), & \boldsymbol{x} \in \Omega, t \geqslant 0 ; & (a) \\
\nabla T \cdot \mathbf{n}=0, & \text { on } \partial \Omega ; & (b) \\
T(\boldsymbol{x}, 0)=T_{0}(\boldsymbol{x}), & \boldsymbol{x} \in \Omega . & (c)
\end{array}\right.
$$

From the basic existence and uniqueness theorems for PDE's, and, under some mild regularity conditions on the boundary of $\Omega$ and on the initial conditions, $T_{0}(\boldsymbol{x}),(\mathbf{P})$ has a unique solution $T(\boldsymbol{x}, t), \boldsymbol{x} \in \Omega, t \geqslant 0$. So, $T$ exists and it is well defined. The Neuman boundary conditions correspond to the condition that the domain is fully isolated from external heat.

\subsection{Thermodynamic Consistency Analysis}

Of course, it is expected that all the temperature solutions, $T(\boldsymbol{x}, t)$, of the heat equation are globally consistent with the first and the second laws of thermodynamics, at all times, independently, of the initial conditions $T_{0}(\boldsymbol{x})$ and of the particular shape of the domain $\Omega$. Although, this result may be well known, for the sake of comparison with the analysis to be presented in Section 4, we state, and explicitly prove the global consistency, below.

We make use of the thermodynamical framework presented in Section 2 . First, let us determine the thermodynamic tuple $\Pi_{\mathbf{P}}=[\mathcal{T}, \mathfrak{e}, \mathfrak{s}, \mathfrak{q}]$ associated to heat equation problem $(\mathbf{P})$. In this case the temperature field $\mathcal{T}$ is the temperature solution $T$, so we only need to determine the remaining fields: $\mathfrak{e}, \mathfrak{q}$ and $\mathfrak{s}$. The identification of these fields is straightforward by noticing that the heat equation 10 models the temperature for the linear conduction 
material, described in Section 2.3. As a consequence, the tuple $\Pi_{\mathbf{P}}$ is of the form given in Eq. (9):

$$
\Pi_{\mathbf{P}}=\bar{\Pi}(T)=[T, \overline{\mathfrak{e}}(T), \overline{\mathfrak{s}}(T), \overline{\mathfrak{q}}(T)]
$$

where $\overline{\mathfrak{e}}, \overline{\mathfrak{s}}, \overline{\mathfrak{q}}$ are given in Eq. (6). Now, we are ready to prove the global consistency of the exact solutions $T(\boldsymbol{x}, t)$.

\subsection{Consistency Analysis with respect to the First Law}

First, let us prove global consistency with respect to the first law of thermodynamics. This is done in the following theorem

Theorem 3.1. All solutions $T(\boldsymbol{x}, t)$ of the heat equation $(\mathbf{P})$ are globally consistent with respect to the first law of thermodynamics.

Proof. Since we are dealing with a fully isolated system (due to the boundary conditions), we need to prove that the thermodynamical tuple $\Pi_{\mathbf{P}}=\bar{\Pi}(T)$ associated to the solutions of the heat equation $(\mathbf{P})$ always satisfies the thermodynamic statement given in Eq. (5)(a):

$$
\int_{\Omega} \rho \dot{\mathfrak{e}} d v=0
$$

The proof goes as follow. From the expression of $\mathfrak{e}$ given in Eq. (11), we have:

$$
\int_{\Omega} \rho \dot{\mathfrak{e}} d v=\int_{\Omega} \rho \frac{\partial \overline{\mathfrak{e}}(T)}{\partial t} d v=\int_{\Omega} \rho c_{v} \dot{T} d v
$$

where in the last equality we have used Eq. (8)(a). Now, using that $T$ satisfies Eq. (10)(a), we have that:

$$
\int_{\Omega} \rho \dot{\varepsilon} d v=\int_{\Omega} \nabla \cdot(\kappa \nabla T) d v
$$

Finally, using divergence theorem on the RHS of the equation above, we have that:

$$
\int_{\Omega} \rho \dot{\mathfrak{e}} d v=\int_{\partial \Omega} \kappa \nabla T \cdot \mathbf{n} d \sigma
$$

Finally, we prove the desired statement (12) by noticing that the RHS term of Eq. (15) is zero, because of the boundary condition (10)(b). 


\subsection{Consistency Analysis with respect to the Second}

Let us prove that the exact solutions $T$ are also globally consistent with respect to the second law of thermodynamics 1 . This is done in the following theorem.

Theorem 3.2. All solutions $T(\boldsymbol{x}, t)$ of the heat equation $(\mathbf{P})$ are globally consistent with the second law of thermodynamics.

Proof. We need to prove that the thermodynamical tuple $\Pi_{\mathbf{P}}$ associated to the solutions of $(\mathbf{P})$ always satisfies the global statement of the second law of thermodynamics defined in Eq. (5)(b), that is to say, we need to prove that:

$$
\int_{\Omega} \rho \dot{\mathfrak{s}} d v \geqslant 0
$$

Proof goes as follows. From the expression of specific entropy of $\Pi_{\mathbf{P}}$ given in Eq. (11), we have that

$$
\int_{\Omega} \rho \dot{\mathfrak{s}} d v=\int_{\Omega} \rho \frac{\partial \overline{\mathfrak{s}}(T)}{\partial t} d v=\int_{\Omega} \rho c_{v} \frac{\dot{T}}{T} d v
$$

where in the last equality we have used Eq. (8-(b). Now, using that $T$ satisfies Eq. (10)(a) in the last integral of the equation above, we get that

$$
\int_{\Omega} \rho \dot{\mathfrak{s}} d v=\int_{\Omega} \frac{1}{T} \nabla \cdot(\kappa \nabla T) d v
$$

Using the chain rule in the integrand on the RHS of the equation above, we obtain that:

$$
\int_{\Omega} \rho \dot{\mathfrak{s}} d v=\int_{\Omega} \nabla \cdot\left(\frac{1}{T} \kappa \nabla T\right) d v+\int_{\Omega} \frac{1}{T^{2}} \kappa \nabla T \cdot \nabla T d v
$$

Noticing that the second term in the RHS of the equation is always greaterequal than zero and using divergence theorem on the first term of the RHS, we get that:

$$
\int_{\Omega} \rho \dot{\mathfrak{s}} d v \geqslant \int_{\partial \Omega} \frac{1}{T} \kappa \nabla T \cdot \mathbf{n} d \sigma
$$

Finally, we prove the desired statement by noticing that the RHS term of the equation above is zero, because of the boundary condition (10)(b).

\footnotetext{
${ }^{1}$ It must be pointed out that, the diffusion problem $(\mathbf{P})$ has been derived from the first law of thermodynamics (2)(a), $\rho \dot{\mathfrak{e}}=-\nabla \cdot \mathfrak{q}$ and the expressions (6) of specific energy (i) and heat flux (ii). The second law has not been used at all. This means that satisfaction of the second law is not self-evident and it should be checked a posteriori.
} 


\section{Thermodynamic Consistency Analysis of CGFE Discretizations of the Heat Equation}

In the previous section, we proved that all exact solutions $T(\boldsymbol{x}, t)$ of the heat equation $(\mathbf{P})$, are globally consistent with, both, the first and the second law of thermodynamics. In this section, we will obtain discrete solutions $T^{h}(\boldsymbol{x}, t)$ of problem $(\mathbf{P})$, generated with the CGFE method, and we will check if they satisfy the same desired properties.

\subsection{The CGFE Discrete Solutions of the Heat Equation $(\mathbf{P})$}

Obtaining the exact solutions of continuum problems is usually a complex if not impossible task. Discretization methods come in our help and allows us to obtain accurate approximations to the original problem. This is what occurs, for example, in the case of heat equation problem $(\mathbf{P})$, defined in Section 3.1. Obtaining all exact solutions $T(\boldsymbol{x}, t)$ is impossible, and the CGFE method allows us to obtaing explicit approximated discrete solutions $T^{h}(\boldsymbol{x}, t)$, which in the limit tend to $T(\boldsymbol{x}, t)$. We describe next the set of equations that allow us to obtain $T^{h}(\boldsymbol{x}, t)$. The CGFE will be used as spatial discretization method, while time $t$ will remain unchanged 2 . The finite element technique, is based in defining a interpolated approximation, $T^{h}(\boldsymbol{x}, t)$, to the exact solution $T(\boldsymbol{x}, t)$. The discrete approximation is given by the following expression:

$$
T^{h}(\boldsymbol{x}, t)=\sum_{j=1}^{n} \varphi_{j}(\boldsymbol{x}) T_{j}(t)
$$

where $\varphi_{j}(\boldsymbol{x})$ denotes the nodal basis function associated to node $j$ located at position $\boldsymbol{x}_{j}$ in space, $n$ denotes the number of nodes of the mesh $\mathrm{M}^{h}$, and where, $T_{j}(t)=T^{h}\left(\boldsymbol{x}_{j}, t\right)$ denotes the corresponding nodal temperature at node $j$. As usual, assume the mesh if made of a spatial partition of the material domain $\Omega$ formed by $m$ arbitrary non-overlapping elemental subdomains $\Omega^{e}$ whose union cover the total domain: $\Omega=\bigcup_{e} \Omega^{e}$. Whenever possible the explicit dependence on $t$ will be dropped, so $T_{j}(t)$ and $\dot{T}_{j}(t)$ will be simply denoted by $T_{j}$ and $\dot{T}_{j}$, respectively. Also, let $\boldsymbol{T}$ denote the column vector of nodal temperatures $T_{j}$, and let $\dot{\boldsymbol{T}}$ denote the column vector of nodal temperature-rates $\dot{T}_{j}$, at any given time $t$. With the above definitions, application of the CGFE spatial discretization on the original

\footnotetext{
${ }^{2}$ The approach is usually known as semi-discretization
} 
diffusion problem $(\mathbf{P})$ leads to the following discretized problem $\left(\mathbf{P}^{h}\right)$ :

$$
\left(\mathbf{P}^{h}\right)\left\{\begin{array}{l}
\text { Find } T^{h}(\boldsymbol{x}, t), \text { where } \\
T^{h}(\boldsymbol{x}, t)=\sum_{j=1}^{n} \varphi_{j}(\boldsymbol{x}) T_{j}(t), \\
\dot{T}^{h}(\boldsymbol{x}, t)=\sum_{j=1}^{n} \varphi_{j}(\boldsymbol{x}) \dot{T}_{j}(t), \\
\text { with } T_{j}(t) \text { and } \dot{T}_{j}(t) \text { found from: } \\
\mathbb{M} \dot{\boldsymbol{T}}=-\mathbb{K} \boldsymbol{T}, \\
\boldsymbol{T}(0), \text { given, (initial condition) }
\end{array}\right.
$$

Note that time-differentiation of Eq. (22) (a) leads to the temperature rates $\dot{T}^{h}(\boldsymbol{x}, t)$ given in Eq. (22)(b). In Eq. $(22)(\mathrm{c}), \mathbb{M}=\left[\mathbb{M}_{i j}\right]$ is the mass matrix and $\mathbb{K}=\left[\mathbb{K}_{i j}\right]$ is the diffusion matrix, respectively. Let us recall that semidiscrete Eq. (22)(c), $\mathbb{M} \dot{\boldsymbol{T}}=-\mathbb{K} \boldsymbol{T}$, is obtained using standard finite element procedure (see [7, for example) which consists in using Eqs. (22)(a)-(b) into the weighted weak form of the original diffusion equation Eq. $(10)(\mathrm{a})$ :

$$
\int_{\Omega} \varphi_{i} \rho c_{v} \dot{T} d v=\int_{\Omega} \varphi_{i} \nabla \cdot(\kappa \nabla T) d v
$$

In arriving to $\mathbb{M} \dot{\boldsymbol{T}}=-\mathbb{K} \boldsymbol{T}$, it has also been used that the boundary integral, appearing during integration by parts of the diffusive term in the weak form, vanishes according to Eq. (10)(b). 3. This procedure leads to the following standard expressions for the mass and difussion matrices

$$
\begin{gathered}
\mathbb{M}_{i j}=\rho c_{v} \int_{\Omega} \varphi_{i} \varphi_{j} d v \\
\mathbb{K}_{i j}=\kappa \int_{\Omega} \nabla \varphi_{i} \cdot \nabla \varphi_{j} d v
\end{gathered}
$$

Like problem $(\mathbf{P})$, problem $\left(\mathbf{P}^{h}\right)$ is well defined and there exist a unique temperature solution field $T^{h}(\boldsymbol{x}, t)$ for $t \geqslant 0$.

For future use, the following two finite element identities are mentioned:

$$
\begin{gathered}
\sum_{i}^{n} \varphi_{i}(\boldsymbol{x})=1 \\
\sum_{i}^{n} \mathbb{K}_{i j}=0
\end{gathered}
$$

\footnotetext{
${ }^{3}$ Note that for this boundary condition, no temperature fixations are needed along the nodes forming the body's boundary $\partial \Omega$.
} 
Eq. 26) is the partition of unity property of basis functions. The second identity can be derived from the definition of the stiffness matrix $\mathbb{K}$ (Eq. 25) and the use of Eq. (26). Also, note that Eq. (22)(c), $\mathbb{M} \dot{\boldsymbol{T}}=-\mathbb{K} \boldsymbol{T}$, can alternatively be written as:

$$
\dot{\boldsymbol{T}}=-\mathbb{H} \boldsymbol{T} \quad \text { where } \quad \mathbb{H}=\mathbb{M}^{-1} \mathbb{K}
$$

$\mathbb{H}$ is called the effective diffusion matrix of the system. It is worth mentioning that matrices $\mathbb{M}$ and $\mathbb{K}$ can be computed by assembly of elemental matrices:

$$
\mathbb{M}_{i j}=\sum_{e}^{m} \mathbb{M}_{i j}^{(e)}, \quad \mathbb{K}_{i j}=\sum_{e}^{m} \mathbb{K}_{i j}^{(e)},
$$

where

$$
\mathbb{M}_{i j}^{(e)}=\rho c_{v} \int_{\Omega^{e}} \varphi_{i} \varphi_{j} d v, \quad \mathbb{K}_{i j}^{(e)}=\kappa \int_{\Omega^{e}} \nabla \varphi_{i} \cdot \nabla \varphi_{j} d v
$$

Then, $\mathbb{M}^{(e)}=\left[\mathbb{M}_{i j}^{(e)}\right]$ and $\mathbb{K}^{(e)}=\left[\mathbb{K}_{i j}^{(e)}\right]$ conform the elemental mass and the elemental diffusion matrices, respectively.

For future use, we provide the expressions of the elemental matrices $\mathbb{M}^{(e)}$ and $\mathbb{K}^{(e)}$ for piecewise linear elements in $1 \mathrm{D}$ and $2 \mathrm{D}$ cases. In the $1 \mathrm{D}$ case, the 'elements' are segments, as the one shown in Fig. 1a, the elemental matrices are given by

$$
\mathbb{M}^{(e)}=\frac{\rho c_{v} h_{e}}{6}\left[\begin{array}{ll}
2 & 1 \\
1 & 2
\end{array}\right], \quad \mathbb{K}^{(e)}=\frac{\kappa}{h_{e}}\left[\begin{array}{rr}
1 & -1 \\
-1 & 1
\end{array}\right]
$$

where $h_{e}$ is the length of the element. In the $2 \mathrm{D}$ case, the elements are triangles (see Fig. 1b). The general expressions for $\mathbb{M}^{(e)}([8]$, pp. 473) and $\mathbb{K}^{(e)}([9],[10])$ in this case are

$$
\begin{gathered}
\mathbb{M}^{(e)}=\rho c_{v} \frac{\sigma^{e}}{12}\left[\begin{array}{ccc}
2 & 1 & 1 \\
1 & 2 & 1 \\
1 & 1 & 2
\end{array}\right] \\
\mathbb{K}^{(e)}=\frac{\kappa}{2}\left[\begin{array}{ccc}
\cot \left(\alpha_{2}\right)+\cot \left(\alpha_{3}\right) & -\cot \left(\alpha_{3}\right) & -\cot \left(\alpha_{2}\right) \\
-\cot \left(\alpha_{3}\right) & \cot \left(\alpha_{1}\right)+\cot \left(\alpha_{3}\right) & -\cot \left(\alpha_{1}\right) \\
-\cot \left(\alpha_{2}\right) & -\cot \left(\alpha_{1}\right) & \cot \left(\alpha_{1}\right)+\cot \left(\alpha_{2}\right)
\end{array}\right]
\end{gathered}
$$

where $\sigma^{e}$ is the triangle's area and where $\alpha_{i}$ denote the triangle's inner angle associate to node $i$. 


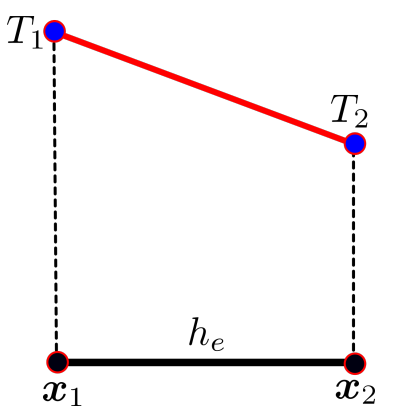

(a) $1 \mathrm{D}$ element

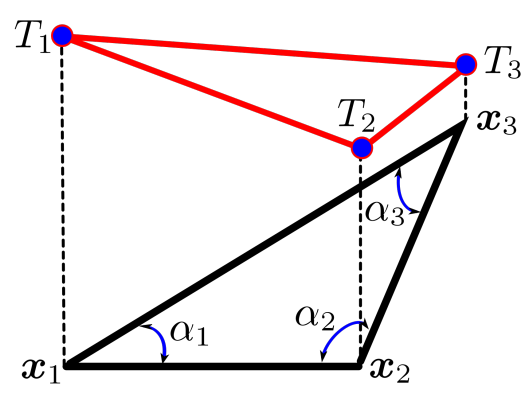

(b) $2 \mathrm{D}$ triangular element

Figure 1: Linear elements in 1D and 2D

\subsection{Thermodynamic Consistency Analysis}

The question posed here is if the temperature solutions $T^{h}(\boldsymbol{x}, t)$ generated by the CGFE method, and defined in problem $\left(\mathbf{P}^{h}\right)$, are globally thermodynamically consistent. In order to answer this question, we need to reconstruct the thermodynamic tuple $\Pi_{\mathbf{P}^{h}}=[\mathcal{T}, \mathfrak{e}, \mathfrak{s}, \mathfrak{q}]$ associated to $\left(\mathbf{P}^{h}\right)$. The reconstruction is straightforward. The thermodynamical tuple $\Pi_{\mathbf{P}^{h}}$ is equal to $\Pi_{\mathbf{P}}$ except that the temperature field, is not longer the exact solution $T(\boldsymbol{x}, t)$ but its CGFE approximation $T^{h}(\boldsymbol{x}, t)$, so:

$$
\Pi_{\mathbf{P}^{h}}=\bar{\Pi}\left(T^{h}\right)=\left[T^{h}, \overline{\mathfrak{e}}\left(T^{h}\right), \overline{\mathfrak{s}}\left(T^{h}\right), \overline{\mathbf{q}}\left(T^{h}\right)\right]
$$

Now, having the corresponding tuple $\Pi_{\mathbf{P}^{h}}=\bar{\Pi}\left(T^{h}\right)$ well defined, we can test for global thermodynamic consistency of the CGFE solutions.

\subsubsection{Consistency Analysis with respect to the First Law}

Let us prove that the discrete solutions $T^{h}(\boldsymbol{x}, t)$ are globally consistent with respect to the first law of thermodynamics. This is done in the following theorem.

Theorem 4.1. All discrete CGFE solutions $T^{h}(\boldsymbol{x}, t)$ of the heat equation (P) are globally consistent with respect to the first law of thermodynamics.

Proof. We need to prove that the termodynamical tuple $\Pi_{\mathbf{P}^{h}}=\bar{\Pi}\left(T^{h}\right)$ produced by the solutions $T^{h}$ always satisfies the global statement of the first law of thermodynamics, Eq. (5)(a). In other words, we need to prove that:

$$
\int_{\Omega} \rho \dot{\mathfrak{e}} d v=0
$$


for $\Pi_{\mathbf{P}^{h}}=\bar{\Pi}\left(T^{h}\right)$.

The proof goes as follows. From the expression of specific energy $\mathfrak{e}$ of $\Pi_{\mathbf{P}^{h}}$ given in Eq. (34), we have:

$$
\int_{\Omega} \rho \dot{\mathfrak{e}} d v=\int_{\Omega} \rho \frac{\partial \overline{\mathfrak{e}}\left(T^{h}\right)}{\partial t} d v=\int_{\Omega} \rho c_{v} \dot{T}^{h} d v
$$

where, for the last equality, we have used Eq. (8-(a). Now, using the expression for $\dot{T}^{h}$ given in Eq. (22)(b), we get that

$$
\int_{\Omega} \rho \dot{\mathfrak{e}} d v=\sum_{j}\left(\int_{\Omega} \rho c_{v} \varphi_{j} d v\right) \dot{T}_{j}
$$

Using the partition of unity property $\sum_{i} \varphi_{i}=1$, we get:

$$
\int_{\Omega} \rho \dot{\mathfrak{e}} d v=\sum_{i} \sum_{j}\left(\int_{\Omega} \rho c_{v} \varphi_{i} \varphi_{j} d v\right) \dot{T}_{j}=\sum_{i} \sum_{j} \mathbb{M}_{i j} \dot{T}_{j}
$$

where in the last equality we have used the definition of mass matrix $\mathbb{M}$. Now, using Eq. (22)(c), we get that:

$$
\int_{\Omega} \rho \dot{\mathfrak{e}} d v=\sum_{i} \sum_{j} \mathbb{K}_{i j} T_{j}=\sum_{j}\left(\sum_{i} \mathbb{K}_{i j}\right) T_{j}
$$

Finally, we prove the desired statement, Eq. (35), by noticing that the last term of the equations above is zero, because of the stiffness matrix property condition (27).

Note that the above theorem is valid for all solutions $T^{h}$ independently of the initial conditions and the mesh. The theorem proves that all solutions $T^{h}$ conserve total energy.

\subsubsection{Consistency Analysis with respect to the Second Law}

Now, we address the problem of determining if the CGFE solutions are globally consistent with respect to the second law of thermodynamics. Contrary of what might be conjectured we will prove that not all obtained CGFE solutions are globally consistent with the second law of thermodynamics. In order to prove this we shall build particular cases where the second law statement, $\int_{\Omega} \rho \dot{\mathfrak{s}} d v \geqslant 0$, is violated. In order, to perform this demonstration, which is the main aim of this paper, we shall first present some derived formulas that will allow us to compute the necessary expression $\int_{\Omega} \rho \dot{\mathfrak{s}} d v$ from the CGFE solutions $T^{h}(\boldsymbol{x}, t)$. These formulas are presented in the following lemma. 
Lemma 4.1. At any time $t$, the rate of change of total entropy $\dot{\mathcal{S}}^{h}=\int_{\Omega} \rho \dot{\mathfrak{s}} d v$ associated to the CGFE solutions, $T^{h}(\boldsymbol{x}, t)$, can be computed as sum of elemental contributions using the following general formula:

$$
\dot{\mathcal{S}}^{h}(t)=\int_{\Omega} \rho \dot{\mathfrak{s}}(\boldsymbol{x}, t) d v=\sum_{e} \dot{\mathcal{S}}^{e}(t)
$$

where:

$$
\dot{\mathcal{S}}^{e}(t)=\rho c_{v} \int_{\Omega^{e}} \frac{\sum_{j^{e}}^{n^{e}} \varphi_{j^{e}}(\boldsymbol{x}) \dot{T}_{j^{e}}(t)}{\sum_{k^{e}}^{n^{e}} \varphi_{k^{e}}(\boldsymbol{x}) T_{k^{e}}(t)} d v
$$

where $k^{e}$ and $j^{e}$ denote local indices of the basis functions $\varphi_{j}$ that are active in element $e$ and where $n^{e}$ denote the total number of these functions, and where, $T_{j}$ and $\dot{T}_{j}$ denote the nodal temperature and nodal temperature rates associated to those local indices, respectively.

Proof. Recall that the thermodynamic tuple $\Pi_{\mathbf{P}^{h}}=\bar{\Pi}\left(T^{h}\right)$ associated to the solutions $T^{h}$ of $\left(\mathbf{P}^{h}\right)$ is given in Eq. (34). From the expression of specific entropy $\mathfrak{s}$ of such equation, we have:

$$
\dot{\mathcal{S}}^{h}=\int_{\Omega} \rho \dot{\mathfrak{s}} d v=\int_{\Omega} \rho \frac{\partial \overline{\mathfrak{s}}\left(T^{h}\right)}{\partial t} d v=\int_{\Omega} \rho c_{v} \frac{\dot{T}^{h}}{T^{h}} d v
$$

where in the last equality we have used Eq. (8-(b). Now, using the expressions for $T^{h}$ and $\dot{T}^{h}$ given in Eqs. (22)(a-b) in the last term of the equations above we get that:

$$
\dot{\mathcal{S}}^{h}(t)=\rho c_{v} \int_{\Omega} \frac{\dot{T}^{h}(\boldsymbol{x}, t)}{T^{h}(\boldsymbol{x}, t)} d v=\rho c_{v} \int_{\Omega} \frac{\sum_{j} \varphi_{j}(\boldsymbol{x}) \dot{T}_{j}(t)}{\sum_{k} \varphi_{k}(\boldsymbol{x}) T_{k}(t)} d v
$$

In the equations above, we have introduced the function arguments for helping the reader to understand what is being computed. We have also made use that $\rho$ and $c_{v}$ are assumed constants for simplicity. Note from Eq. (43) that computing total entropy rate $\dot{\mathcal{S}}^{h}$ requires the computation of a rational function having basis functions in the numerator and denominator. It is not possible to find an analytical expression for this integral. However, we can procced further and make use of finite element properties to decompose such integral as the sum of elemental entropy-rate contributions, $\dot{\mathcal{S}}^{e}$, along the mesh elements $\Omega^{e}$. Then, performing this decomposition, Eq. (43) can be re-written as:

$$
\dot{\mathcal{S}}^{h}(t)=\sum_{e} \dot{\mathcal{S}}^{e}(t)
$$


where

$$
\dot{\mathcal{S}}^{e}(t)=\rho c_{v} \int_{\Omega^{e}} \frac{\dot{T}^{h}(\boldsymbol{x}, t)}{T^{h}(\boldsymbol{x}, t)} d v=\rho c_{v} \int_{\Omega^{e}} \frac{\sum_{j} \varphi_{j}(\boldsymbol{x}) \dot{T}_{j}(t)}{\sum_{k} \varphi_{k}(\boldsymbol{x}) T_{k}(t)} d v
$$

Then, keeping only the nodal basis functions $\varphi$ that are active in each element ' $e$ ', it follows that Eq. (45) can be rewritten as Eq. (41), which completes the proof.

Next, analytical expressions for the entropic elemental contributions $\dot{\mathcal{S}}^{e}$ will be obtained for the popular cases of piecewise linear basis functions in $1 \mathrm{D}$ and 2D, i.e., for segments and triangular elements. This formulae will be presented in the following two lemmas.

Lemma 4.2. At any time $t$, the rate of change of elemental entropy $\dot{\mathcal{S}}^{e}$, for piecewise linear $1 D$ elements can be computed from the following formula:

$$
\left\{\begin{array}{l}
\dot{\mathcal{S}}^{e}=\rho c_{v} h_{e} \frac{N}{D} \\
\text { where } \\
\text { if } T_{1} \neq T_{2} \\
N=\left(T_{1}-T_{2}\right)\left(\dot{T}_{1}-\dot{T}_{2}\right)+\left(T_{2} \dot{T}_{1}-T_{1} \dot{T}_{2}\right) \log \left[T_{2} / T_{1}\right] \\
D=\left(T_{1}-T_{2}\right)^{2} \\
\text { if } T_{1}=T_{2} \\
N=\dot{T}_{1}+\dot{T}_{2} \\
D=2 T_{1}
\end{array}\right.
$$

where $h_{e}$ is the lenght of element $e$, and where, $T_{1}, T_{2}$ and $\dot{T}_{1}, \dot{T}_{2}$ are the nodal temperatures and the nodal rates of the $1 D$ element, at such time $t$, as shown in Fig. 1 a.

Proof. In the 1D case, the general formula for $\dot{\mathcal{S}}^{e}$, given in Eq. (41), becomes:

$$
\dot{\mathcal{S}}^{e}=\rho c_{v} \int_{h_{e}} \frac{\varphi_{1}(x) \dot{T}_{1}+\varphi_{2}(x) \dot{T}_{2}}{\varphi_{1}(x) T_{1}+\varphi_{2}(x) T_{2}} d x=\rho c_{v} \int_{x_{1}}^{x_{2}} \frac{\varphi_{1}(x) \dot{T}_{1}+\varphi_{2}(x) \dot{T}_{2}}{\varphi_{1}(x) T_{1}+\varphi_{2}(x) T_{2}} d x
$$

where local indices associated to element $e$ are used (see Fig. 1a). Now, the integral along the segment $\left[x_{1}, x_{2}\right]$ can be mapped into a master segment, $[0,1]$, of unit lenght having local coordinates $u$ with vertices at $u_{1}=0$ and $u_{2}=1$, so Eq. 477 can be re-written as:

$$
\dot{\mathcal{S}}^{e}=\rho c_{v} h_{e} \int_{0}^{1} \frac{(1-u) \dot{T}_{1}+u \dot{T}_{2}}{(1-u) T_{1}+u T_{2}} d u
$$


Note that the nodal functions $\varphi_{1}$ and $\varphi_{2}$, in local coordinates $u$, become $\varphi_{1}=1-u$ and $\varphi_{2}=u$, respectively. After analytical integration of the RHS of Eq. 48, one obtains that $\dot{\mathcal{S}}^{e}$ can be computed from Eq. 46). This completes the proof.

Lemma 4.3. At any time $t$, the rate of change of elemental entropy $\dot{\mathcal{S}}^{e}$, for piecewise linear triangular elements can be computed from the following formula:

$$
\dot{\mathcal{S}}^{e}=\rho c_{v} 2 \sigma^{e} \frac{N_{1} \dot{T}_{1}+N_{2} \dot{T}_{2}+N_{3} \dot{T}_{3}}{D}
$$

where

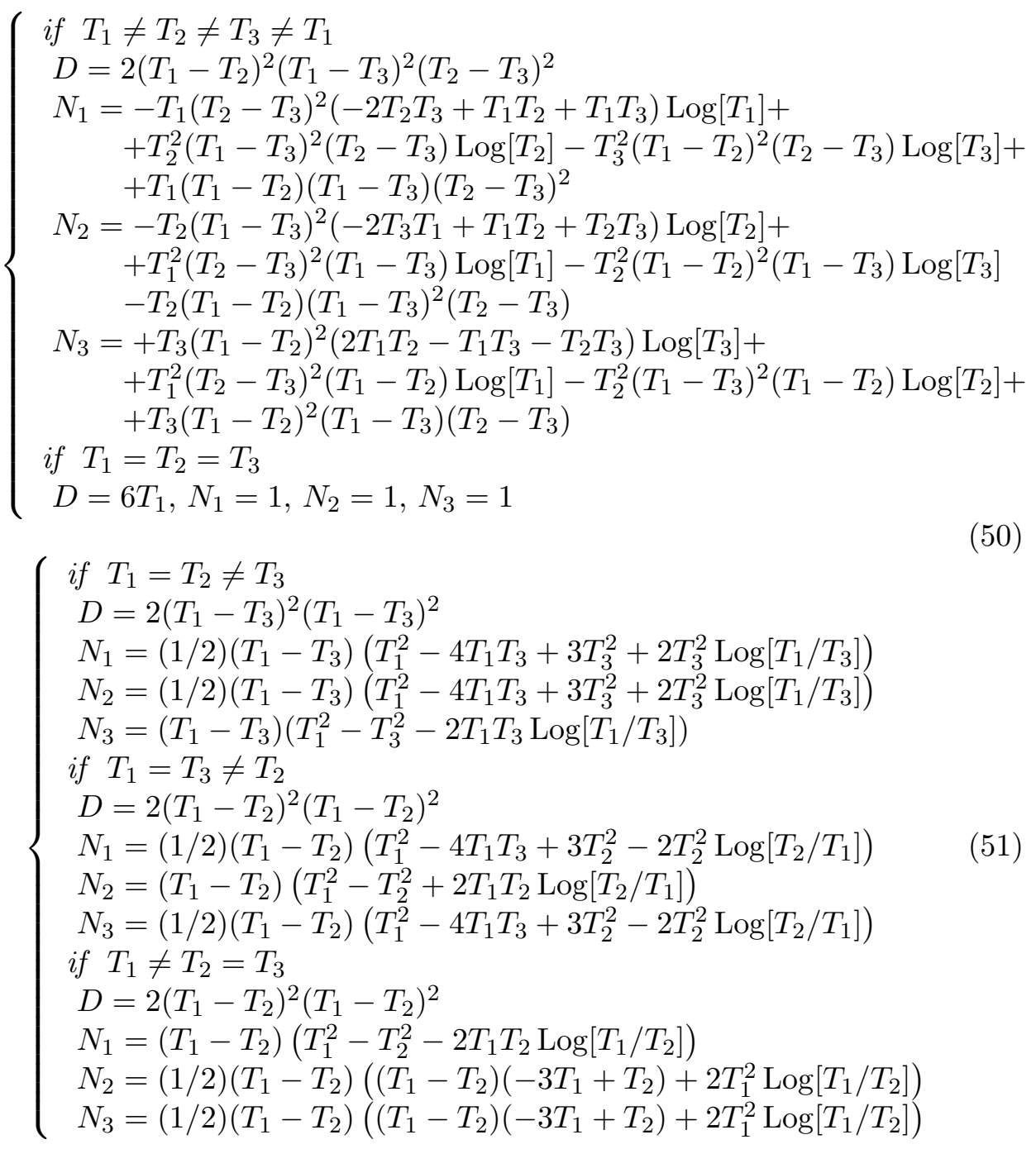


where $h_{e}$ is the lenght of element e, and where, $T_{1}, T_{2}$ and $\dot{T}_{1}, \dot{T}_{2}$ are the nodal temperatures and the nodal rates of the $1 D$ element, at such time $t$, as shown in Fig. 1 a.

Proof. In the 2D case, with triangular elements, Eq. (41) becomes:

$$
\dot{\mathcal{S}}^{e}=\rho c_{v} \int_{\sigma^{e}} \frac{\varphi_{1} \dot{T}_{1}+\varphi_{2} \dot{T}_{2}+\varphi_{3} \dot{T}_{3}}{\varphi_{1} T_{1}+\varphi_{2} T_{2}+\varphi_{3} T_{3}} d a
$$

where, again, local elemental indices are used and where $\varphi_{i}$ is the shape function of node $i$, located at vertex $\boldsymbol{x}_{i}$ of the triangular element, as shown in Fig. 1b), and where, $\left\{T_{i}, \dot{T}_{i}, i=1,2,3\right\}$ denote the corresponding nodal temperatures and the nodal temperature-rates, at time $t$, at such nodal points. For the sake of simplicity assume that the local elemental index numbering is chosen so "node 1 " has the minimun nodal temperature, that is to say: $T_{1}=\min \left(T_{1}, T_{2}, T_{3}\right)$. Note also that $T$ is absolute temperature, so one must have that $T_{1}, T_{2}, T_{3}>0$. Mapping the above integral into a master triangular element having local coordinates $(u, v)$ and vertices $(0,0),(0,1)$ and $(1,0)$, and using the expressions of the shape functions in terms of these local coordinates, one has that the elemental entropy can be computed as:

$$
\dot{\overline{\mathcal{S}}}^{e}=\rho c_{v} 2 \sigma^{e} \int_{0}^{1} \int_{0}^{1-u} \frac{(1-u-v) \dot{T}_{1}+u \dot{T}_{2}+v \dot{T}_{3}}{(1-u-v) T_{1}+u T_{2}+v T_{3}} d u d v
$$

where $\sigma^{e}$ is the area of the triangular element $\Omega^{e}$. Then the above integral can be written as:

$$
\dot{\overline{\mathcal{S}}}^{e}=\frac{\rho c_{v} 2 \sigma^{e}}{T_{1}} \int_{0}^{1} \int_{0}^{1-u} \frac{(1-u-v) \dot{T}_{1}+u \dot{T}_{2}+v \dot{T}_{3}}{(1-u-v)+u\left(T_{2} / T_{1}\right)+v\left(T_{3} / T_{1}\right)} d u d v
$$

From Eq. (54), it follows that except by a scaling factor, the elemental entropy rate depends on the relative values of nodal temperatures. After analytical integration of the RHS of Eq. (54), one obtains that $\dot{\mathcal{S}}^{e}$ can be computed from Eq. (47). This completes the proof.

From the above Lemmas, it follows that, at any time $t$, the total entropy rate $\dot{\mathcal{S}}^{h}=\int_{\Omega} \rho \dot{\mathfrak{s}} d v$ of any discrete finite element solution $T^{h}(\boldsymbol{x}, t)$ can be computed by summation of the elemental contributions $\dot{\mathcal{S}}^{e}(t)$ calculated using Eq. (46) for 1D linear elements and Eq. (49) for linear triangular elements. 4

\footnotetext{
${ }^{4} \mathrm{~A}$ similar procedure can be used to compute total entropy rates for other types of elements.
} 
Now, the above lemmas will be used to build simple examples which will prove that the CGFE method is not entropically consistent. The examples will prove that the CGFE method can generate discrete solutions $T^{h}(\boldsymbol{x}, t)$ that violate the global statement of the second law of thermodynamics given in Eq. (5(b), which asserts that total entropy must always satisfy that $\int_{\Omega} \rho \dot{\mathfrak{s}} d v \geqslant 0$.

\subsubsection{Failure Examples 1D Case}

Consider the case of heat conduction in a one-dimensional material bar of unit lenght $L=1$ whose end points are located at $x=0$ and $x=1$, as the one shown in red in Fig. 2, Assume for simplicity that the bar is made of a material with unit properties $\rho, c_{v}, \kappa=1$. Assume the bar is fully isolated from the exterior. Suppose the bar is discretized by a mesh of 5 equal elements and 6 nodes, and, assume the initial temperature distribution in the bar is given by $\boldsymbol{T}=\left[T_{1}, T_{2} \ldots, T_{6}\right]^{\top}=[90,10,1,1,10,90]^{\top}$, as shown in Fig. 2 . The instantaneous vector of nodal temperature rates $\dot{\boldsymbol{T}}$ is determined by the CGFE method through Eq. (22)(c). The rate of change of total entropy $\dot{\mathcal{S}}^{h}$ associated to such evolutionary state can be computed using Eqs. 40 and (46). The resulting value is $\dot{\mathcal{S}}^{h}=-24.67$. This negative value indicates a violation of the second law of thermodynamics (??). This proves that the CGFE is not entropically consistent for linear elements in 1D. It must be pointed out that not all discrete solutions produced by the CGFE method violate the second law, only some of them do. For example, if one runs an experiment where different vectors of nodal temperatures $\boldsymbol{T}$ $=\left[T_{1}, T_{2} \ldots, T_{6}\right]^{\top}$ are generated by choosing the nodal temperatures from the set of four possible values $T_{i}=\{1,10,40,90\}, i=1: 6$, only 5 values of $\boldsymbol{T}$ (out of the 4096 cases) will generate a temperature evolution that violate the condition $\dot{\mathcal{S}}^{h} \geq 0$. Also, it is important to note that the violation of entropy not necessarily will occur at all times. For example, if the time-evolution of nodal temperatures (see Fig. 3a) is computed for subsequent times for the case of the initial condition $\boldsymbol{T}$ shown in Fig. 2, it can be observed from Fig. 3b that the entropy rate will violate the second law of thermodynamics only at the first times of the simulation. Of course, such violation will have an impact in the future temperature behavior. At least one anomalous behavior can be detected in the predicted evolution of nodal temperatures shown in Fig. 3a: at initial times, the nodes that have the minimum temperature $\left(T_{i}=T_{\min }=1, i=3,4\right)$, instead of showing an increase in their temperatures (heat should flow from warmer to colder regions), they show a non-physical reduction of their temperatures below $T_{\min }$ (indicated by the dotted red line in Fig. 3a). As a consequence, one 


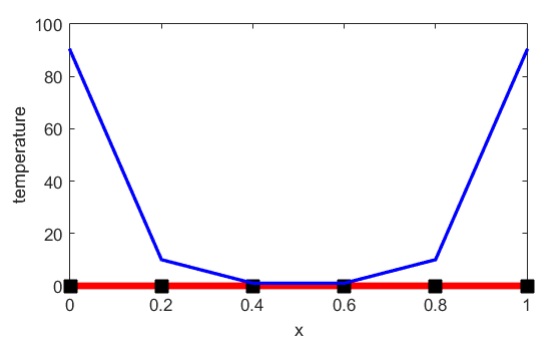

Figure 2: Heat conduction in an isolated 1D bar shown in red color. The bar is discretized by 5 elements with an initial temperature distribution shown in blue color

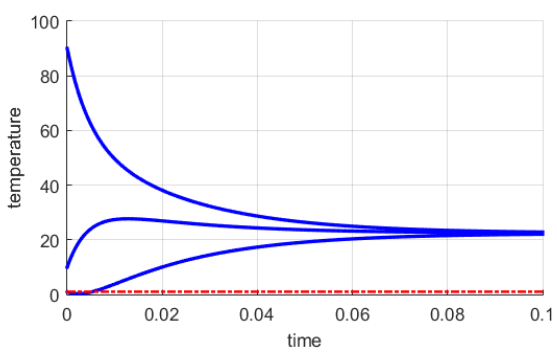

(a) Evolution of nodal temperatures $\boldsymbol{T}(t)$

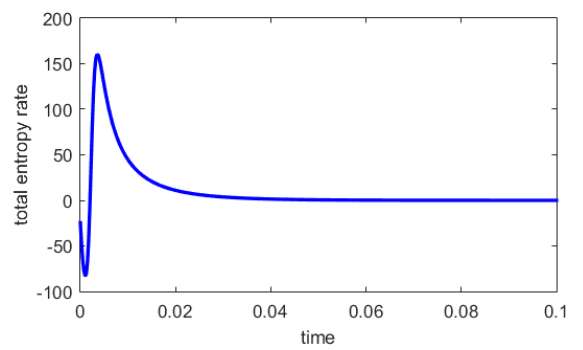

(b) Evolution of total entropy rate $\dot{\mathcal{S}}^{h}(t)$

Figure 3: Temperature and total entropy rate time-evolution predicted by the CGFE method in an isolated 1D bar for the initial temperature distribution shown in Fig. 2

may speculate that the negative values of $\dot{\mathcal{S}}^{h}$ may indicate the presence of non-physical, reversed, heat flows occurring inside the domain due to the discretization process. This is in agreement with a recent result [4].

\subsubsection{Failure Examples 2D Case}

Consider the following heat conduction problem in a body with quadrangular shape, as the one shown in Fig. ??. Assume the body is fully isolated: it does not received nor give up heat from the exterior. For simplicity, assume that the body is made of a material with unit properties $\rho, c_{v}, \kappa=1$. Assume the body is discretized by a mesh of 12 equal triangular elements and 12 nodes, as shown in Fig. ??. The triangular elements conforming the mesh have inner angles given by: $\alpha_{1}=20 \mathrm{deg}$, $\alpha_{2}=150 \mathrm{deg}$ and $\alpha_{3}=10 \mathrm{deg}$. The nodal coordinates $\boldsymbol{x}_{i}=\left(x_{i}, y_{i}\right)$ are given by $\boldsymbol{x}=[(0,0) ;(1.4251,0) ;(2.8502,0) ;(2.4308,1.4034) ;(3.8559,1.4034)$; (5.2810,1.4034); (4.8616, 2.8069); (6.2867,2.8069); (7.7118, 2.8069); (7.2924, $4.2103) ;(8.7175,4.2103) ;(10.1426,4.2103)]$. Note that the vector of nodal 
temperatures is defined by $\boldsymbol{T}=\left[T_{1}, T_{2} \ldots, T_{12}\right]^{\top}$. Given a vector $\boldsymbol{T}$, the vector of nodal temperature rates $\dot{\boldsymbol{T}}$ can be found from Eq. (22)(c). With the values of $\{\boldsymbol{T}, \dot{\boldsymbol{T}}\}$, the total entropy rate $\dot{\mathcal{S}}^{h}$ of the discrete solution can be computed from Eqs. (40) and (49). Now, let us run a numerical experiment where values of $\dot{\mathcal{S}}^{h}$ will be computed for different initial temperature states $\boldsymbol{T}$. For this purpose, let us assign each nodal temperature $T_{i}, i=1, . ., 12$ a temperature value of the following set $\{1,10,50\}$, considering all possible permutations of resulting vectors $\boldsymbol{T}$. With 12 nodes there are a total number of $3^{12}=531441$ possible permutations. For each permutation, a value of $\boldsymbol{T}$ is set, $\dot{\boldsymbol{T}}$ can be determined, and $\dot{\mathcal{S}}^{h}$ can be computed to verify that the second law of thermodynamics is satisfied $\left(\dot{\mathcal{S}}^{h} \geq 0\right)$. If CGFE discretizations were fully thermodynamically consistent, such condition should be satisfied for all permutations. The results of the experiment show that, like in the $1 \mathrm{D}$ case, some discrete solutions violate the second law $\left(\dot{\mathcal{S}}^{h}<0\right)$. For the present experiment, 15 entropically inconsistent solutions were detected. They are given in Table 1. The corresponding values of negative entropy rates are shown in the last column. In Fig. ??, the distribution of temperatures in the quadrangular body are shown for the entropically inconsistent case defined in the 12th-row of Table 1 . The experiments proves that the CGFE method is not consistent with respect to the second law of thermodynamics for linear triangular elements.

\section{Conclusions}

A thermodynamic consistency analysis of the Continuous Galerkin Finite Element (CGFE) method has been conducted. It is well known that the CGFE method is globally consistent with respect to the the first law of thermodynamics. However, contrary to what one would have expected, in this paper, it is proved that the method is not always globally consistent with respect to the second law of thermodynamics. The global statement of this law imposes that, in an isolated system, total entropy can never decrease. Here, it is shown that the CGFE method can produce discrete solutions that violate such important physical law, when standard piecewise linear elements in 1D and 2D are used. Further investigation need to be done to answer the question if non-standard versions of the CGFE method can be used, under which, global consistency with respect to the second law can be guarantee. A possible road would be to explore if the use of lumped mass matrices may help in the recovery of second law compatibility. Numerical experiments performed with linear elements in $1 \mathrm{D}$ would indicate that second law's violation might be successfully removed by using such matrices. 


\begin{tabular}{|cccccccccccc|c|}
\hline$T_{1}$ & $T_{2}$ & $T_{3}$ & $T_{4}$ & $T_{5}$ & $T_{6}$ & $T_{7}$ & $T_{8}$ & $T_{9}$ & $T_{10}$ & $T_{11}$ & $T_{12}$ & $\dot{\mathcal{S}}$ \\
\hline 1 & 1 & 1 & 1 & 1 & 1 & 1 & 1 & 10 & 1 & 10 & 50 & -16.4754 \\
1 & 1 & 1 & 1 & 1 & 1 & 1 & 1 & 10 & 10 & 10 & 50 & -17.4644 \\
1 & 1 & 1 & 1 & 1 & 10 & 1 & 10 & 50 & 10 & 50 & 50 & -13.6147 \\
1 & 1 & 1 & 1 & 1 & 10 & 10 & 10 & 50 & 10 & 50 & 50 & -0.3109 \\
1 & 1 & 1 & 1 & 1 & 10 & 10 & 10 & 50 & 50 & 50 & 50 & -2.7110 \\
1 & 1 & 10 & 1 & 10 & 50 & 10 & 50 & 50 & 50 & 50 & 10 & -1.2388 \\
1 & 1 & 10 & 1 & 10 & 50 & 10 & 50 & 50 & 50 & 50 & 50 & -0.2620 \\
10 & 50 & 50 & 50 & 50 & 10 & 50 & 10 & 1 & 10 & 1 & 1 & -1.2388 \\
50 & 10 & 1 & 10 & 1 & 1 & 1 & 1 & 1 & 1 & 1 & 1 & -16.4754 \\
50 & 10 & 10 & 10 & 1 & 1 & 1 & 1 & 1 & 1 & 1 & 1 & -17.4644 \\
50 & 10 & 10 & 10 & 1 & 1 & 1 & 1 & 10 & 10 & 10 & 50 & -1.9806 \\
50 & 50 & 10 & 50 & 10 & 1 & 10 & 1 & 1 & 1 & 1 & 1 & -13.6147 \\
50 & 50 & 10 & 50 & 10 & 10 & 10 & 1 & 1 & 1 & 1 & 1 & -0.3109 \\
50 & 50 & 50 & 50 & 10 & 10 & 10 & 1 & 1 & 1 & 1 & 1 & -2.7110 \\
50 & 50 & 50 & 50 & 50 & 10 & 50 & 10 & 1 & 10 & 1 & 1 & -0.2620 \\
\hline
\end{tabular}

Table 1: Nodal temperature configurations $\boldsymbol{T}$ producing violation of the second law of thermodynamics $\left(\dot{\mathcal{S}}^{h}<0\right)$

For linear triangular elements in 2D, this does not seem to be enough. In this case, meshes with special types of triangular shapes might be necessary. The authors think that the issue discussed in this paper might be connected with the observation that CGFE discretizations violate, nodally, the Clausius's Postulate of the second law which has been reported in a recent work [4]. In this sense, the global inconsistency may be a measure of the existence of non-physical reversed heat flows produced by the discretization procedure. Finally, it must be pointed out that the mathematical background presented here is general and could be used to explore the thermodynamic consistency of other types of elements, such as quadrangular or tetrahedral elements, as well, as other variants of finite element formulations.

\section{References}

[1] A. Limache, S. Idelsohn, R. Rossi, E. Oñate, The violation of objectivity in Laplace formulations of the Navier-Stokes equations, International Journal for Numerical Methods in Engineering 54 (2007) 639-664.

[2] A. Limache, P. Sanchez, L. Dalcin, S. Idelsohn, Objectivity tests for Navier-Stokes simulations: the revealing of non-physical solutions pro- 
duced by Laplace formulations, Computer Methods in Applied Mechanics and Engineering 197 (2008) 1703-1759.

[3] T. J. R. Hughes, G. Engel, L. Mazzei, M. G. Larson, The continuous galerkin method is locally conservative, Journal of Computational Physics 163 (2000) 467-488.

[4] A. Limache, S. Idelsohn, On the issue that finite element discretizations violate, nodally, Clausius's postulate of the second law of thermodynamics, Advanced Modeling and Simulation in Engineering Sciences 3 (13) (2016) 1-18.

[5] M. Gurtin, E. Fried, L. Anand, The Mechanics and Thermodynamics of Continua, Cambridge University Press, 2010.

[6] C. Truesdell, Rational Thermodynamics, Springer-Verlag, 1984.

[7] M. Larson, F. Bengzon, The Finite Element Method: Theory, Implementation and Applications, Vol. 10 of Texts in Computational Science and Engineering, Springer, 2013.

[8] O. Zienkiewicz, R. Taylor, The Finite Element Method - Fifth Edition, Vol. 1, Butterworth-Heinemann, 2000.

[9] A. Draganescu, T. F. Dupont, L. R. Scott, Generalized local maximum principles for finite-difference operators, Math. of Comp. 27 (124) (2004) 685-718.

[10] I. Faragó, R. Horváth, S. Korotov, Discrete maximum principle for linear parabolic problems solved on hybrid meshes, Applied Numerical Mathematics 53 (2005) 53 (2005) 249-264. 